\title{
Title The Big Bang of the Brain
}

\section{First Author ${ }^{1}$ Hugo Lagercrantz}

Affiliation Karolinska Institute,

Address, Astrid Lindgren Children's Hospital, 17176 Stockholm

Country Sweden

E-mail:Hugo.Lagercrantz@ki.se

\section{Another Author}

Affiliation

Address, Country

E-mail: another.author@pos.it

Here you can write the abstract for your paper.

Hector Rubinstein was a very open-minded person. It was possible to discuss anything with him on politics, literature and science. He and Helen invited us often to their house meeting leading physicists. I was impressed how they talked about big bang, dark energy, the string theory etc. As a neuroscientist and child doctor I am mainly concerned with more daily problems of patient care. But with Hector making of the brain, expansion of the human cortex, birth and the first breaths became nearly as eternal questions as those he was dealing with. Just a few months before he died he invited me to give a lecture and Albanova. Therefore I tried to apply a perspective from his point of view on brain development. Many neuroscientists seem to have a more biochemical approach dealing mainly with DNA, RNA, transcription factors and neurotransmitters. However, I think a physical approach imaging the function of the brain is more important to understand what it is all about.

9-11, 201

Speaker 


\section{Introduction}

The quest of the origin of the universe out of nothing and the emergence of life are the two most fundamental stages of the creation. But a third quest of similar dignity is how the human brain and mind was formed, which is essential for the reflexion of ourselves but also of the first two questions [1]. We talk often about the big bang of the universe, but not so often about the "big bang" of making the brain - how one hundred billion neurons are formed and connected. There are actually about the same number of neurons in the brain as stars in the Milky way.

The human brain is born two weeks after conception. Before that there is only a blob with undifferentiated cells. But at fourteen days after conception, a primitive streak emerges, the beginning of a head and tail axis. Under the primitive streak a solid rod of cells is formed which is called the notochord. This structure functions like a conductor of an orchestra, instructing what the cells should do. In this way the blob becomes a three-dimensional structure with a head and tail.

This process is called gastrulation and can be regarded as the most important event during life [2]. Otherwise we would remain wormlike organisms.

The notochord plays an important role conducting the fate of the cells to become motor neurons, i.e. nerve cells responsible for muscular movements. This is mediated by the sonic hedge-hog protein, which seems to have an essential role in many of the early processes of brain formation[3].

The primitive streak is thus transformed to a nervous streak, which will become neural groove. This grove will be closed from the middle, like closing a zipper upwards and downwards.

\section{Sonic goes ballooning}

The upper end of the nerve tube will become the brain by swelling. It is like blowing up an oval balloon. Three swellings will emerge, which will form the hollows or ventricles of the brain which will be filled with cerebrospinal fluid. This process is initiated by a special protein made by the sonic hedgehog gene, which is an important gene in the making of the primitive brain [3]. If the effect of the sonic hedge-hog protein is blocked, there will be no swellings and the nerve cord will remain a thread like in worms. The 
sonic hedge hog gene is named after some figures in a video game. This gene is also responsible for the development of wings in insects, legs in mammals and the differentiation of some nerve cells to motor neurons, ie the nerve cells controlling muscular movements. It is said that this gene is promiscious by forming a number of loose connections when convenient. Thus a question of "timing and spacing".

\section{Neurons - the atoms of the mind They are round in the young brain and have few processes according to Ramon y Cajal}
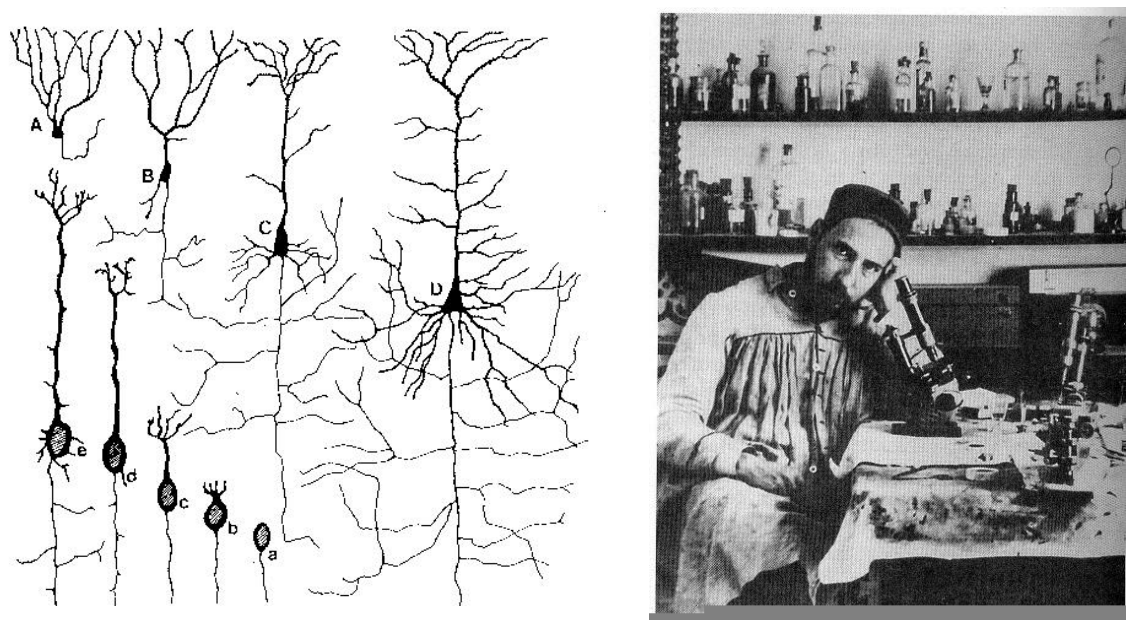

Fig.1

\section{200000 new nerve cells per minute}

Nerve cells prolipherate at a remarkable high speed between the third and fifth months of fetal life. 200,000 new neurons are formed each minute originating from the inner walls of the cavities (ventricles ) of the brain. The source for the production of the nerve cells are the layers of stem cells. Stem cells are undifferentiated cells which can become any type of cell from nerve and muscle cells to blood corpuscles.

Such a stem cell is expelled from the layer and divide. One of the daughter cells become specialized to a nerve cell and can then not return to become any other type of cell. On 
the other hand the second daughter cell will remain stem cell, turn back to the layer and be able to start a new cycle. About 20 cycles take place resulting in the production of one hundred billion of nerve cells. Then this burst of new cells stops and is not assumed to continue after birth possibly except in some areas like in the centre for smell and cerebellum.

\section{0 new nerve cells/min}

\section{Hammarberg 1896 Caviness 2008}
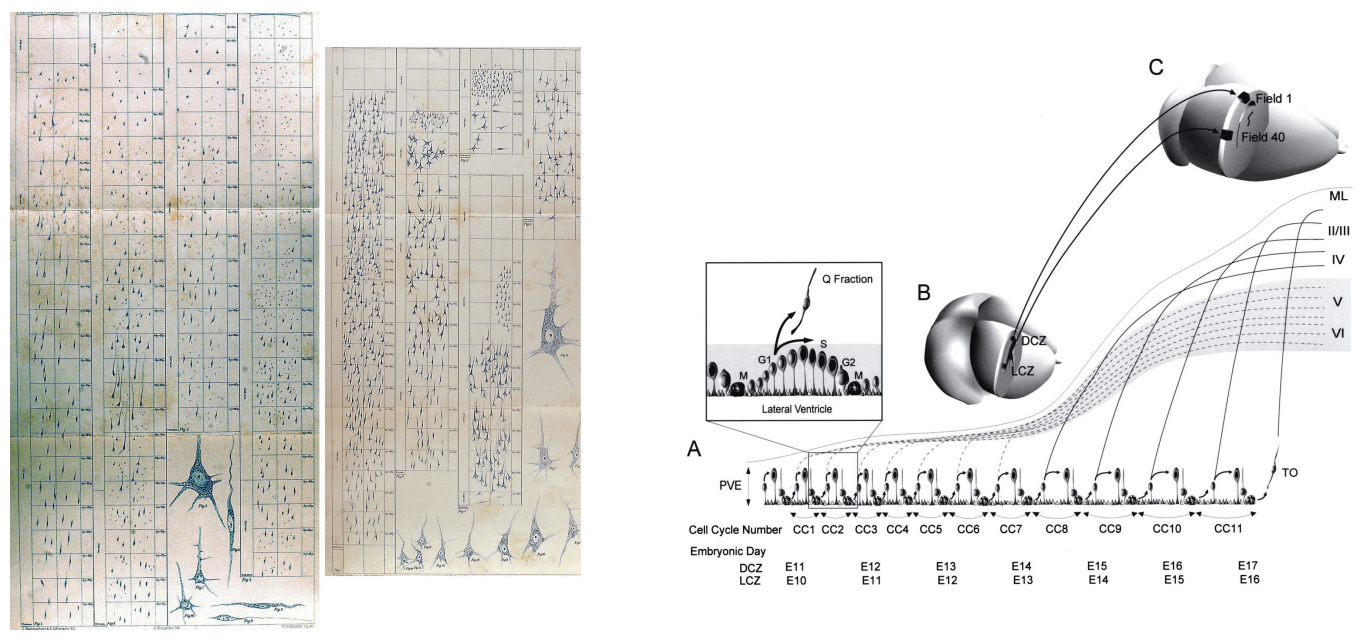

Fig. 2

How do we know this? In one classical British study it was demonstrated that the content of new DNA in the brain peaks between the third and fifth month of aborted human fetuses. Another evidence is that children who had been exposed to strong radiation became microcephalic when they were exposed during this time of fetal life, but not before or after. This was found by studying the effects of the tragic events of the atomic explosions in Hiroshima and Nagasaki.

Further evidences have been presented by the Croatian-American neuroscientist Pasco Rakic. He had studied medicine and Belgrad and written a thesis on brain development based on the investigation of aborted fetuses, which was easy to obtain during the 
Communist era. He then went to the United States, where he performed some fundamental studies on brain development in monkeys. By administration of a a radioactive substance (thymidine) which is incorporated in the DNA, when new cells are formed by division he could demonstrate that nearly all nerve cells are formed before birth in monkeys. It is hard to believe that it would be different in the human fetus, who has a much longer fetal life [4].

Therefore it has been stated that nearly all nerve cells are formed before birth in the human in reviews and textbooks. It had nearly become a dogma, until the agronomist Dr Fernando Nottebohm originally from Argentine stood up at a conference at in New York, claiming that new nerve cells can be formed in the adult brain. He had demonstrated that new nerve cells are formed in the singing center of canarian male birds, and the number of new nerve cells are related to the repertoire. This was a revolutionary finding against the established dogma that almost all nerve cells in the brain are formed during early life. Nottebohm claimed that new nerve cells can also emerge in the adult human brain. This has actually been confirmed leading to a hype to develop new treatments of Parkinson's and Alzheimer's diseases, by stimulating proliferation of nerve cells in damaged areas of the brain.

In the audience there were several professors from Harvard and Yale who shook their heads, Pasco Rakic stood up and said that something like: 'If new nerve cells can be made during adult life I would be able to get rid of my Croatian accent." There is probably some meaning that we retain the same nerve cells in the brain throughout life. The audience laughed.

But after the discovery of the production of new nerve cells in the adult canarian birds, an intensive search formation of the new neurons in the adult human brain began. A Swedish doctor met a collegue in the emergency who happened to treat patients with laryngeal cancer with a nucleotide analogue, the same substance which is used to label DNA to date the formation of nerve cells. They therefore decided to analyze this substance in the brains of these patients who usually died soon in spite of the treatment. They then discovered about 500 new nerve cells in the hippocampus of these 50-70 year old patients . Other scientists also reported the formation of new nerve cells in the adult primate brain. 
Thus, there are some evidences that we can exchange brain cells in the brain during whole life like we do in the skin and the gut. However, is it possible to exchenage neurons in the cerebral cortex, where we believe most ofour consciosness and personaly are localized?

No, this seems less likely. Even if some new nerve cells can emerge during adult life in lower parts of the brain, no new neurons are formed in the cerebral cortex. This has recently been deomonstrated by an ingeneous technique developed by the Swedish neuroscientist Jonas Frisén. It is based on the fact that nucelar tests in the 1950s and early 1960 s produced a dramatic elevation of ${ }^{14} \mathrm{C}$ levels in the atmosphere due to the frequent atomic tests performed above the ground at that time. After the tests were performed underground the levels decreased due to dispersion in the oceans etc. Like archeologists they could then date, when the nerve cells are formed, since this is reflected by the incorporation of the ${ }^{14} \mathrm{C}$ in the DNA. They could then clearly demonstrate that no new cells were formed in the cerebral cortex after birth in contrast to other parts of the brain and the body. Thus we can conclude that we have the same neurons in the cortex throughout life, although several of them disappear but they are not substituted by new ones [5]. Thus we can retain old memories and our personality until old age.

\section{The invation of nerve cells}

The nerve cells migrate towards the top and the sides of the head along thin threads of glia - the supporting cells in the brain. These threads function like ropes hanging in a a scaffold. The nerve cells are climbing along these ropes. In the beginning they form a subplate, from which they enter the cortex from about the 22nd-24th gestational week. There are also nerves migrating along a horizontal axis [4]. We can talk about an invasion of nerve cells which form the cortex. This invasion is much more impresive in the human fetus than in other mammals. Probably some mutation occurred a couple of million years ago leading to this explosion of nerve cells invading the tip of the head. This may explain why the human cortex is so much more expanded than in the monkey and can harbour for example symbolic thinking and language.Even if monkeys can 
communicate with each other, the repertoire of signs and words is much more limited than in the human.

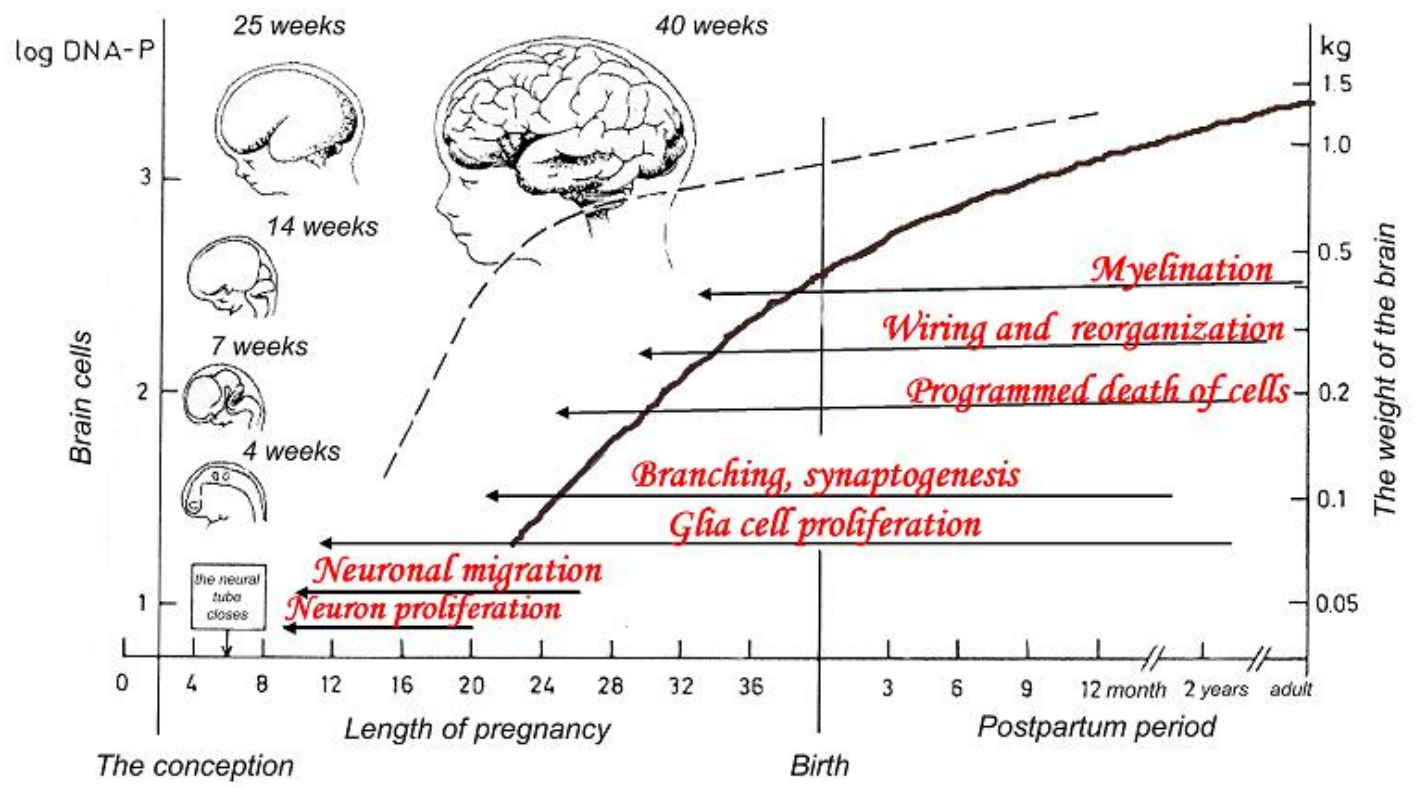

\section{Fig. 3}

\section{Synaptic cross-talk}

The nerve cells are naked from the beginning and cannnot communicate. This was demonstrated by the famous Spanish anatomist Ramon y Cajal more than 100 years ago.The next step is that they develop branches, the main one is the axon and several smaller ones are called dendrites. Synapses connect the nerve cells with each other. Every nerve cell has $1000-10,000$ synapses. They begin to be formed slowly alrady in the sixth to 8 th gestational week but then more rapidly from about the 20th week. The synaptogesesis peaks in the human brain between the first and third year of life, when more than up to one million synapses are made every second. This is at the time when the brain is like a magnet sucking in all new words it is exposed to. Words we learn during childhood like other memories become stored in the synapses. The synaptogenesis continues at a very high rate during whole childhood to level off during 
adolescence. After puberty it is difficult to learn a new language without accent. But we can learn new languages and a lot of other things even as adults, since synaptogenesis continues until old age, although it becomes more and more difficult when we become older (see ref [6].

\section{Use it or lose it}

All the billions of nerve cells with trillions of branches and synapses look like a jungle towards the end of the fetal life. Different parts of the brain are connected by several windling pathways. There are connections between areas where all kinds of sensory inputs are processed for example between the areas for vision and hearing. Theoretical the fetus can hear the flash of lightning and see the thunder. This phenomena is called synaesthesia and can remain after birth. Thus some children can can get special visual impressions when they hear certain sounds or feel special smells.

\section{The brain is not a computer, it is a jungle}

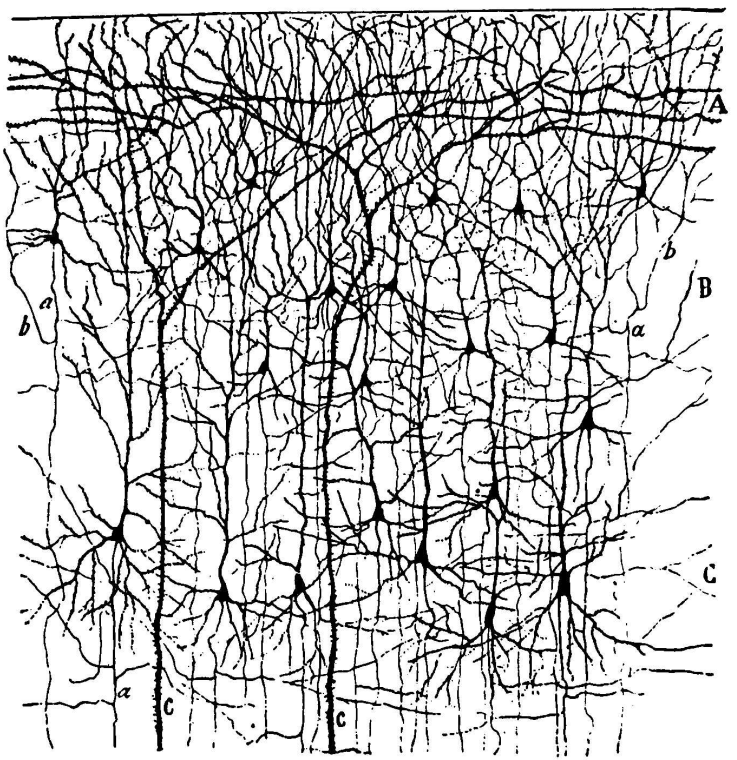

Fig. 4 
The immature brain is like old Paris with a number of small windling streets and gates. There is a redundancy of nerve cells and their connections. But fortunately there are natural mechanisms to organize this jungle. Like Eugéne Haussmann demolished several houses to build the big boulevards, billions of nerve cells and their connections disappear by apoptosis or programmed cell death. This is an important process during development in general, for example it is involved in the disappearance of the web between the fingers and toes. There are certain suicidal genes, which are activated. This occurs also in the brain. Nearly half of all the hundred billion of nerve cells dissappear before birth. To some extent this is genetically determined, but it is also affected by environmental mechanisms. Nerves which are stimulated for example from the sensory organs thrive and develop more branches and synapses. Nerves which do not receive any stimulation disappear. "Cells which fire together wire together, and those which don't won't" or "Use it or lose it".

It is well-known that infants who are born with eye cataract must be operated as soon as possible, otherwise they become blind. The immature brain must be stimulated by visual impressions at an early stage to learn how to see. We talk about a critical period or window for opportunity when the brain must be stimulated to promote the survival of the most approriate nerve connections and elimination of the unnecessary ones. "To learn is to eliminate!" $[7,8]$

\section{The Dark energy of the brain}

About $50 \%$ of all the blood glucose i consumed by the newborn brain compared with $20 \%$ in the adult. To what kind of activity is this source of energy used? Remarkably littte is used for solving specific problem for example a mathematical problem. Most of the glucose seem to be used by the spontaneous activity of the brain, which may correspond to the inner monologue of the brain. This spontaneous neuronal firing is also called resting activity, which is an inadequate term. The brain does not ever seem to be at rest, even if the activity is lower during dreamless sleep and anaesthesia. It can be recorded with functional magnetic resonans (fMRI) which measures the blood oxygen levels-dependent signals or BOLD, which seem to be related to nervous activity. It has also been called the dark energy of the brain, a metaphore borrowed from astrophysics the unseen energy representing the mass of most of the universe [9]. 
The functional role of this spontaneous activity is not evident. By mathematical tools originating from graph theory the networks have been revealed and cortical hubs identified. In adults the hubs were predominantly found in association cortex and prefrontal cortex indicating that the activity is involved in "To retain what no longer is, to anticipate what as yet is not..." (Bergson). This suggests that it is related to consciousness. On the other hand the hubs of motor, sensory, auditory and visual cortex seem to dominate in the newborn infant, suggesting that the infant is more living in the present moment[10] .

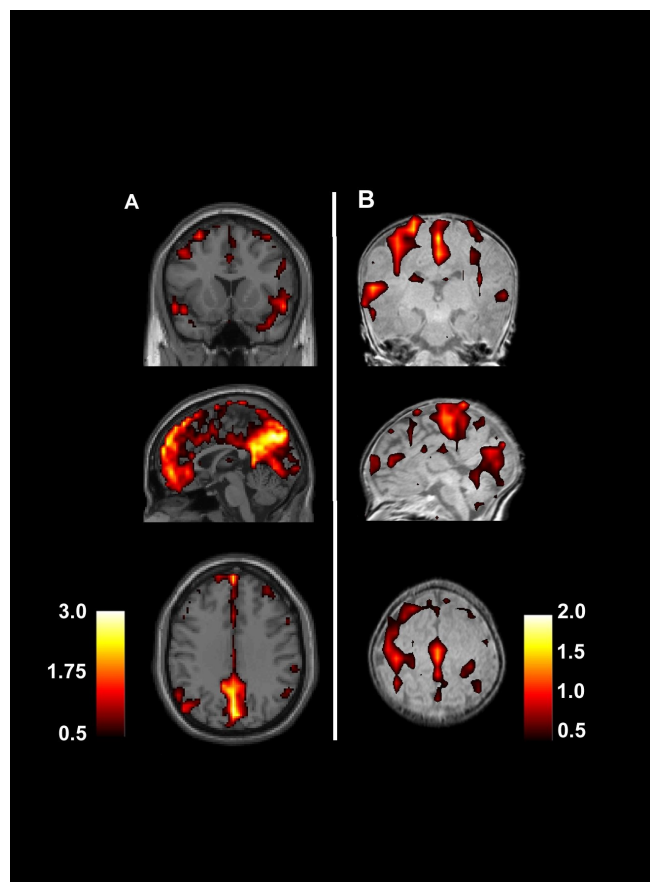

\section{"The inner} monologue" Spontaneous resting activity in the infant $(A)$ and adult brain (B)

Fransson, Lagercrantz et al Cerebral Cortex 2010

Fig. 5

\section{Myelination}

The impuls traffic is fairly slow in the fetal brain. To increase the speed of the neve impulses, they are provided with nerve sheaths, which consist of a special fat. It is like the isolation material around electric copper wires. But the sheat is interrupted at several sites or nodes. The nerve impuls can then jump between these nodes and in this way increase the speed. Myelination is a very important process starting already around the 
23rd week with the largest so called pyramidal nerve cells. But myelination continues throughout whole childhood. Thinner nerves are myelinated much later. The last nerve cells which are myelinated are the small so called internerons in the forebrain. This may occur late during adolescence, even up to 30 years. This late myelination of the nerves in the frontal lobe is important for executive actions taking place in this part of the brain. It may explain why adolescents may have difficulties to make correct executive decisions. It is possibly the cause why young people more often are involved in traffic accidends than adults.

Myelination can be observed with magnetic resonce imaging in the form of white matter. The thickness of white matter increases in the cortex during childhood and adolescense.

\section{Nature and Nurture}

To what extent is brain development determined by the genes (nature) or the environment (nurture)? When the human genome was mapped there was a strong emphasis on nature, i.e, a number of behavioral problems were postulated to be mainly due to genes. Genes increasing the risk for attention deficit hyperactivty diorders (ADHD), intelligens, shyness, depression, criminality, alcoholism were identified making big headlines in the news media. Although there are certainly some genes which increase the risk for developing various behavoral properties, environmental factors also play an important role. Mice pups which are neglected by the dams have difficulties when tested for cognitive functions as adults. They are also more sensitive to stress . This is not very surprising, but the new finding is that the genome is affected during sensitive periods in early life. It is then important to open or close the right segments of the DNA spiral by methylation and demethylation [11]. Similar changes in the genome as in the neglected mice have been detected in humans committing suicide, who had been maltreated as children.

Brain development is certainly initially determined by specific genes forming the neural tube, nerve cells and their migration. Also the branching of the nerves and synaptogenesis is originally genetically determined. However from the third trimester ie the third part of fetal life, intrinsic as well as exrinsic nervous activity play a more important role for make the synapses and the neuronal circuits. This is even more 
important after birth. It is hard to believe that the structure of the brain with 100 billions of nerve cells, each one with 1000-10000 synapes can be determined in detail by only 22,000 genes.

Thus to-day we do not talk about nature or nurture, heredity or environment but insead on nature and nurture. We can maybe say that the making of our brain and mind is determined to $100 \%$ by nature but also to $100 \%$ by nurture.

In Nature a new figure was presented of the DNA spiral including "the other strand" representing the cultural heredity, which is of particular importance for human development [12].

Acknowledgement: This article is a revised version of an article in French: La fabrication du cerveau published in Science\&Humaine 2010

1. Koch, C., The quest for consciousness: a neurobiological approach, ed. R.a.C. Publishers2004, Eaglewood, Colorado. 429.

2. Wolpert, L., Principles of Development 1998, NY: Oxford University Press.

3. Britto, J., D. Tannahill, and R. Keynes, A critical role for sonic hedgehog signaling in the early expansion of the developing brain. Nat Neurosci, 2002. 5(2): p. 103-10.

4. Rakic, P., Specification of cerebral cortical areas. Science, 1988. 241(4862): p. 170-6.

5. Nowakowski, R., Stable neuron numbers form cradle to grave. Proceedings of the National Academy of Science, 2006. 103(33): p. 12219-12220.

6. $\quad$ Lagercrantz H, H.M., Ment L, Peebles DM (eds), The newborn brain (2nd ed)2010: Cambridge University Press. 412.

7. Changeux, J.P., Neuronal Man1997, Princeton, NJ: Princeton University Press.

8. Changeux, J.P., P. Courrege, and A. Danchin, A theory of the epigenesis of neuronal networks by selective stabilization of synapses. Proc Natl Acad Sci U S A, 1973. 70(10): p. 2974-8.

9. Raichle, M., The Brain's dark energy. Scientific American, 2010. March: p. 2833.

10. Fransson, P., Adén, U., Blennow, M., Lagercrantz, H., The functional architecture of the infant brain as revealed by resting state fMRI. Cerebral Cortex, 2011. 21: p. 145-154.

11. Kappeler, L., Meaney, M.J., Epigenetics and parental effects. Bioassays, 2010. 32: p. 818-827.

12. Hayden, E.C., The other strand. Nature, 2009. 457: p. 776-779.

[1] f Mock conference PoS(MC2000)002. 